\title{
Who should perform the rhinopharyngeal swab in COVID-19 positive patients?
}

\author{
Armando De Virgilio et al. ${ }^{1}$
}

${ }^{1}$ Affiliation not available

April 28, 2020

\section{Dear Editor,}

The COVID-19 infection can be diagnosed from a variety of upper and lower respiratory sources including the oropharynx (OP), nasopharynx (NP), sputum, and bronchial fluid [1-3]. In general the most sensitive detection of COVID-19 is obtained by the collecting and testing of both upper and lower respiratory samples [4].

However, bronchoscopy is a highly technical procedure requiring advanced diagnostic tools as well as welltrained staff which are not always available. Furthermore the collection of sputum and particularly BAL via bronchoscopy increases the biosafety risk to healthcare workers through the creation of aerosol droplets.

Upper respiratory specimens such as OP and NP swabs are easy to collect especially in limited resource settings. They should be collected within the first few days from the onset of symptoms since RNA positive rates peak in upper respiratory tract specimens at 7-10 days after symptom onset and then they steadily decline [5].

In China during the COVID-19 outbreak, Wang et al reported that oropharyngeal (OP) swabs ( $\mathrm{n}=398)$ were used much more frequently than NP swabs $(\mathrm{n}=8)$. However, the COVID-19 RNA was detected only in $32 \%$ of OP swabs, compared to NP swabs $(63 \%)[4]$.

It appears to be extremely important to properly collect nasopharyngeal swabs reaching the posterior rhinopharyngeal tonsil region. This implies the presence of a regular nasal cavity floor. Some anatomical variants, such as nasal septum deviation, can prevent reaching of the nasopharynx and therefore to collect a proper sample. Numerous studies of nasal septal deviation have revealed a wide range of prevalence [6,7]. In 1978, Gray reported a prevalence of $48 \%$ to $60 \%$ in neonates [6].1 In adults, a recent international study found a prevalence of approximately $90 \%$ [7]. Sooknundun et al. reported a clinically relevant septal deviation prevalence of $15 \%$ to $25 \%$ [8].

Current national and international guidelines do not include any special recommendations in the execution of the rhinopharyngeal swab in patients with documented nasal pathology or in patients in which a bilateral nasal fossa obstacle is encountered. We believe that in these selected cases the ENT support should be mandatory in order to obtain a representative sample. Furthermore the use of endoscopes could be very useful in the direct visualization of obstacles and to guide safely the swab toward the rhinopharynx. This would possibly also reduce the false negative rate which is reported to be more than $30 \%$ [4].

Armando De Virgilio ${ }^{1,2}$, Raul Pellini ${ }^{3}$, Giuseppe Mercante ${ }^{1,2}$, Fabio Ferreli ${ }^{1,2}$, Gerardo Petruzzi ${ }^{3}$, Giuseppe Spriano $^{1,2}$ 
1. Otorhinolaryngology Unit, Humanitas Clinical and Research Center-IRCCS, Viale Manzoni 56, Rozzano (Mi), Italy. 2. Department of Biomedical Sciences, Humanitas University, Via Rita Levi Montalcini, 4, 20090 Pieve Emanuele (MI) Italy. 3. Department of Otolaryngology - Head and Neck Surgery, IRCCS Regina Elena National Cancer Institute, 00144 Rome, Italy.

Corresponding author: Armando De Virgilio, Otorhinolaryngology Unit, IRCCS Humanitas Clinical and Research Centre, via Manzoni 56, 20089 Rozzano (Mi), Italy. Tel.: +39- 028224 8282; Fax: +39- 028224 8282; E-mail: armando.devirgilio@gmail.com

\section{References}

Gaunt ER, Hardie A, Claas EC, et al. Epidemiology and clinical presentations of the four human coronaviruses 229E, HKU1, NL63, and OC43 detected over 3 years using a novel multiplex real-time PCR method. J Clin Microbiol. 2010;48(8):2940-2947. doi:2910.1128/JCM.00636-00610 doi: 10.1128/JCM.00636-10

Charlton CL, Babady E, Ginocchio CC, et al. Practical guidance for clinical microbiology laboratories: viruses causing acute respiratory tract infections. Clin Microbiol Rev. 2019;32(1). doi:00010.01128/CMR.0004200018 doi: 10.1128/CMR.00042-18

Falsey AR, Formica MA, Walsh EE. Simple method for combining sputum and nasal samples for virus detection by reverse transcriptase PCR. J Clin Microbiol. 2012;50(8):2835. doi:2810.1128/JCM.01473-01412 doi: 10.1128/JCM.01473-12

Cheng PK, Wong DA, Tong LK, et al. Viral shedding patterns of coronavirus in patients with probable severe acute respiratory syndrome. Lancet. 2004;363(9422):1699-1700. doi:1610.1016/S0140-6736(1604)1625516257

Loeffelholz MJ, Tang YW. Laboratory diagnosis of emerging human coronavirus infections - the state of the art. Emerg Microbes Infect. 2020 Dec;9(1):747-756. doi: 10.1080/22221751.2020.1745095.

Gray LP. Deviated nasal septum. Incidence and etiology. Ann Otol Rhinol Laryngol Suppl 1978;87(3 Part 3 Suppl 50):3-20.

Mladina R, Cujic E, Subaric M, Vukovic K. Nasal septal deformi- ties in ear, nose, and throat patients: An international study. Am J Otolaryngol 2008;29(2):75-82.

Sooknundun M, Kacker SK, Bhatia R, Deka RC. Nasal septal deviation: Effective intervention and long term follow-up. Int J Pediatr Otorhinolaryngol 1986;12(1):65-72 